\title{
Mitigation of energetic electrons in the magnetosphere by amplified whistler wave under double cyclotron resonances
}

\author{
S. P. Kuo \\ Department of Electrical and Computer Engineering, Polytechnic Institute of New York University, 6 MetroTech Center, \\ Brooklyn, NY 11201, USA
}

Received: 9 July 2008 - Revised: 5 September 2008 - Accepted: 5 September 2008 - Published: 22 October 2008

\begin{abstract}
An optimal approach reducing the population of $\mathrm{MeV}$ electrons in the magnetosphere is presented. Under a double resonance condition, whistler wave is simultaneously in cyclotron resonance with $\mathrm{keV}$ and $\mathrm{MeV}$ electrons. The injected whistler waves is first amplified by the background $\mathrm{keV}$ electrons via loss-cone negative mass instability to become effective in precipitating $\mathrm{MeV}$ electrons via cyclotron resonance elevated chaotic scattering. The numerical results show that a small amplitude whistler wave can be amplified by more than $25 \mathrm{~dB}$. The amplification factor reduces only about $10 \mathrm{~dB}$ with a $30 \mathrm{~dB}$ increase of the initial wave intensity. Use of an amplified whistler wave to scatter $1.5 \mathrm{MeV}$ electrons from an initial pitch angle of $86.5^{\circ}$ to a pitch angle $<50^{\circ}$ is demonstrated. The ratio of the required wave magnetic field to the background magnetic field is calculated to be about $8 \times 10^{-4}$.
\end{abstract}

\section{Introduction}

In the magnetosphere, energetic electrons are trapped by the Earth's magnetic dipole field to undergo a bouncing motion about the geomagnetic equator. Energetic electrons in the $\mathrm{MeV}$ range have a strong impact on passing satellite systems. Satellites are designed to survive a certain amount of radiation (ionizing) dose accumulated during their lifetimes. Unexpected enhancement of the radiation fluxes caused by, for example, very strong solar storms, will significantly increase the total radiation dose to the satellites. Consequently, the radiation damage on active electronics and detectors of satellite systems will accumulate faster than that designed for. As the damage exceeds a threshold level, satellite systems become incapable of performing their mission. It is essential to find

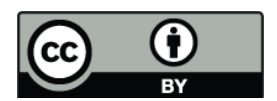

Correspondence to: S. P. Kuo (skuo@duke.poly.edu) ways which can mitigate unexpected radiation enhancement to keep satellite systems less vulnerable.

Whistler waves can be ducted in an L-shell of the magnetosphere to continuously interact with the energetic electrons trapped in the same L-shell. The motions of energetic electrons are adversely affected by the wave fields which scatter some of them into loss cones (Helliwell et al., 1973). Induced electron precipitation (Voss et al., 1984; Arnoldy and Kintner, 1989; Imhof et al., 1994; Pradipta et al., 2007) by whistler waves has been observed. The Doppler shifted electron cyclotron resonance interaction (Kennel and Petschek, 1966; Villalon and Burke, 1991; Albert, 2000) has been suggested to be a likely electron precipitation mechanism. The numerical results show that the electron cyclotron resonance interaction can diffuse energetic electrons, with their initial pitch angles close to the loss cone angle, into the loss cone, via small angle scattering process (Albert, 2000). However, the number of electrons resonant with the wave at a given frequency is small. Moreover, the resonance condition is anisotropic, which makes it difficult to explain the observation of precipitation events occurring simultaneously at geomagnetic conjugate regions due to a single lightning flash (Burgess and Inan, 1990).

The trajectories of trapped energetic electrons in the presence of whistler waves can become chaotic, subject to that the whistler wave intensity (Faith et al., 1996, 1997a, b; Kuo et al., 2004) and/or the initial electron energy (Khazanov et al., 2008) exceed threshold levels. Once chaos occurs, many of electrons can wander into both loss cones. This chaotic (large angle) scattering process precipitates electrons to both loss cones simultaneously. Cyclotron resonance can reduce the threshold wave field for the commencement of chaos in the electron trajectories.

Magnetospheric energetic electrons have an anisotropic velocity distribution, which is potentially unstable to electromagnetic waves (Tsytovich and Stenflo, 1983). Indeed, it has been observed (Helliwell et al., 1980; Helliwell and Inan,

Published by Copernicus Publications on behalf of the European Geosciences Union and the American Geophysical Union. 
1982; Helliwell, 1983) that trapped energetic (keV) electrons in the magnetosphere can significantly amplify whistler waves. The anisotropic relativistic plasma can also excite electromagnetic instability through the electron cyclotron resonance interaction (Tsurutani and Smith, 1974; Nunn et al., 1997; Trakhtengerts, 1999; Trakhtengerts et al., 2004). VLF wave generation by energetic electrons is evidenced by the natural event of chorus (Sazhin and Hayakawa, 1992) occurring in the inner magnetosphere and by the appearance of large amplitude whistler-mode waves in radiation belts (Cattell et al., 2008). The experimental observations also indicate some intrinsic differences between emission and amplification processes. Chorus is basically discrete VLF emissions; on the other hand, the amplitude of the amplified whistler wave oscillates continuously in time.

The relativistic cyclotron resonance condition is a quadratic equation in the electron momentum, thus, there exists a double resonance situation (Kuo et al., 2007), namely, a whistler wave is simultaneously in cyclotron resonance with the $\mathrm{keV}$ electrons and with the $\mathrm{MeV}$ electrons. This suggests an optimal approach, which applies the chaotic scattering process under a double resonance condition, for the control of the population of $\mathrm{MeV}$ electrons trapped in the magnetosphere. This approach first uses keV electrons (having a loss-cone velocity distribution) to energize the incident whistler waves, which become more effective to precipitate $\mathrm{MeV}$ electrons into loss cones, via cyclotron resonance enhanced chaotic scattering.

In this paper, the theoretical basis of this optimal approach is presented and the feasibility of the approach is examined. In Sect. 2, the double cyclotron resonance mechanism is explained. In Sect. 3, the formulation of the nonlinear instability theory is presented; a fifth order differential equation governing the temporal evolution of the whistler field amplitude is derived. Numerical results are also presented. Section 4 devotes to the formulation and analysis of chaotic scattering. An example of chaotic scattering under double cyclotron resonances is given in Sect. 5. Summary is presented in Sect. 6.

\section{Relativistic effect for double cyclotron resonances}

Cyclotron resonance is an effective process to enhance the interaction between wave and charge particles and is essential to whistler wave amplification. Thus the possibility of a double cyclotron resonance situation, under which the wave is simultaneously in cyclotron resonances with $\mathrm{keV}$ electrons for amplification and with $\mathrm{MeV}$ electrons to instigate precipitation, is explored in the following. The Doppler shifted cyclotron resonance condition in the relativistic case is given by

$\omega=\Omega_{0} / \gamma+k P_{z} / \gamma m$

where $\gamma=\left(1+P_{\perp}^{2} / m^{2} c^{2}+P_{z}^{2} / m^{2} c^{2}\right)^{1 / 2}$ is the relativistic factor of the electron, $P_{\perp}=\gamma m v_{\perp}, P_{z}=\gamma m v_{z}$, and $\boldsymbol{k}=\hat{z} k$ is as- sumed; $\omega<\Omega_{0}$ for whistler waves; $\omega$ and $\Omega_{0}=e B_{0} / m$ are the wave frequency and the nonrelativistic electron cyclotron frequency. For a small $\gamma$, i.e., $\omega<\Omega_{0} / \gamma$, the resonant electrons are moving oppositely to the wave propagation direction.

Because of the $\gamma$ dependence, this condition leads to a quadratic equation for $P_{z}$ as

$A P_{z}^{2}+2 B P_{z}+C=0$

where $\quad A=\left(1-\omega^{2} / k^{2} c^{2}\right), \quad B=m \Omega_{0} / k, \quad$ and $\quad C=(m / k)^{2}$ $\left[\Omega_{0}^{2}-\omega^{2}\left(1+P_{\perp}^{2} / m^{2} c^{2}\right)\right]$. This quadratic equation has two real solutions $P_{z}=\left[-B \pm\left(B^{2}-A C\right)^{1 / 2}\right] / A$, subject to the condition $B^{2} \geq A C$. The double solutions suggest that the wave can be simultaneously resonant with two different groups of electrons. The coefficients $A$ and $C$ of the quadratic equation are positive because $\omega / k c<1$ and $\Omega_{0} / \gamma_{1,2}>\omega$ are considered; thus both $P_{z}$ are negative, i.e., the two groups of electrons, which can resonantly interact with the wave, move opposite to the propagation direction of the wave.

\section{Amplification of whistler waves}

We are interested in wave amplification in time; moreover, the amplification mainly proceeds in the region near the magnetic equator, inferred by the common static source region of chorus to be near magnetic equator deduced from correlated chorus elements of different frequency/time characteristics measured by Cluster Wideband Data (WBD) receiver (Breneman et al., 2007). Thus, the formulation can be simplified by assuming a local uniform magnetic field $\mathbf{B}_{0}=B_{0} \hat{z}$. The electron plasma in the magnetosphere consists of three components: 1$)$ cold background $\left.\left(\gamma=\gamma_{0} \sim 1\right), 2\right)$ energetic (in keV range) electrons $\left(\gamma=\gamma_{1}\right)$, and 3 ) very energetic (in $\mathrm{MeV}$ range) electrons $\left(\gamma=\gamma_{2}\right)$. The background electron plasma is nonrelativistic $(\gamma \sim 1)$ and determines the propagation characteristics of the whistler wave, which has the dispersion relation

$\omega=\Omega_{0} c^{2} k^{2} / \omega_{p b}^{2}$

where $\omega_{p b}=\left(4 \pi n_{b} e^{2} / m\right)^{1 / 2} ; n_{b}$ is the background cold electron density.

Energetic electrons in $\mathrm{keV}$ range are weakly relativistic (e.g., $\gamma_{1} \sim 1.1$ for $50 \mathrm{keV}$ electrons) and have considerable density $n_{\varepsilon}$ to amplify whistler waves. These electrons have a loss cone distribution given by

$$
\begin{aligned}
f_{\varepsilon}\left(P_{\perp}, P_{z}\right)= & 2 \pi P_{\perp} F_{\varepsilon}\left(P_{\perp}, P_{z}\right) \\
= & n_{\varepsilon}\left(2 \pi^{-1 / 2} / j !\right)\left(\Delta P_{j \varepsilon}\right)^{-(2 j+3)} P_{\perp}^{2 j+1} \\
& \exp \left[-\left(P_{\perp}^{2}+P_{z}^{2}\right) / \Delta P_{j \varepsilon}^{2}\right] \\
= & n_{\varepsilon} f_{\varepsilon \perp}\left(P_{\perp}\right) f_{\varepsilon z}\left(P_{z}\right)
\end{aligned}
$$

where $\Delta P_{j \varepsilon}=\left[m T_{e \varepsilon} /(1 / 2+j / 3)\right]^{1 / 2} ; \quad T_{e \varepsilon} \gg T_{e b} ;$ subscripts $\varepsilon$ and $b$ stand for "energetic" and "background", respectively. 
The distribution in $P_{z}$ is a Maxwellian having the form

$f_{\varepsilon z}\left(P_{z}\right)=\pi^{-1 / 2} \Delta P_{j \varepsilon}^{-1} \exp \left(-P_{z}^{2} / \Delta P_{j \varepsilon}^{2}\right)$

and the distribution in $P_{\perp}$ has a loss cone form

$$
f_{\varepsilon \perp}\left(P_{\perp}\right)=(2 / j !)\left(\Delta P_{j \varepsilon}\right)^{-(2 j+2)} P_{\perp}^{2 j+1} \exp \left(-P_{\perp}^{2} / \Delta P_{j \varepsilon}^{2}\right)
$$

In the following, an instability process to amplify whistler waves by $\mathrm{keV}$ electrons having a loss cone momentum distribution in the magnetosphere is studied. The relativistic effect, through the Doppler shifted cyclotron resonance (Eq. 1), provides essential nonlinearity for the phase bunching (Kuo and Cheo, 1985) of those electrons in near cyclotron resonant interaction with the wave. The bunched electrons then excite the loss-cone negative mass instability for the wave amplification (Kuo and Lee, 1986). Specifically, the resonant interaction of a whistler wave with a particular group of electrons in the magnetosphere will be formulated. Since these electrons are practically collisionless, a single electron system will be considered to first derive the resonant trajectory equations for a single electron in the wave fields. This derivation is presented in Appendix A. The results are then averaged over the electron's random phase angle (with respect to the wave field) to obtain the collective effect for wave amplification, which is described physically in Appendix B.

The collective result in electron-wave resonance interaction can be demonstrated through a phase average on $\Delta \omega=\Delta \omega_{0}-\left(k^{2} c^{2} / \omega-\omega_{0}\right)\left(\gamma_{1}-\gamma_{10}\right) / \gamma_{1}$, where $\Delta \omega_{0}=\omega-\omega_{0}$ is the initial mismatch frequency and $\omega_{0}=\Omega_{0} / \gamma_{10}-k v_{z 0}$. In carrying out phase average, energy conservation has to be satisfied. The energy conservation equation is given by

$$
\Delta n_{\varepsilon} m c^{2} d\left\langle\gamma_{1}\right\rangle_{\varepsilon} / d t+\varepsilon_{0}\left[\left(1+\varepsilon_{r}\right) / 2\right] d E_{0}^{2}(t) / d t=0
$$

where \langle\rangle$_{\varepsilon}$ represents an average over the initial random phases of those energetic electrons, which are involved in (near) resonant interaction with the wave; $\Delta n_{\varepsilon}=N_{1}$ $N_{2} \cong j^{-1 / 2} n_{\varepsilon}\left[j^{-1 / 2} f_{\varepsilon \perp}^{\prime}\left(P_{\perp 1}\right) f_{\varepsilon z}\left(P_{z 1}\right)-f_{\varepsilon \perp}\left(P_{\perp 1}\right) f_{\varepsilon z}^{\prime}\left(P_{z 1}\right)\right]$ $\times\left(2 \gamma_{1} m \Delta \omega_{0} / k\right)^{3} ; \quad d\left\langle\gamma_{1}\right\rangle_{\varepsilon 2} / d t=-d\left\langle\gamma_{1}\right\rangle_{\varepsilon 1} / d t \quad$ (i.e., $\langle\cos \phi\rangle_{\varepsilon 2} \cong-\langle\cos \phi\rangle_{\varepsilon 1} \quad$ is assumed); $\quad \varepsilon_{r}=1+\omega_{p b}^{2} / \omega \Omega_{0} \quad$ is the dielectric function of background plasma responding to the whistler wave and $\Delta n_{\varepsilon}\left(\ll n_{\varepsilon} \ll n_{b}\right)$ is the net density of energetic electrons transferring energy to the wave through the resonant interaction with the wave. An integration of Eq. (6) leads to

$$
\Delta n_{\varepsilon} m c^{2}\left(\left\langle\gamma_{1}\right\rangle_{\varepsilon}-\left\langle\gamma_{10}\right\rangle_{\varepsilon}\right)=-\varepsilon_{0}\left[\left(1+\varepsilon_{r}\right) / 2\right]\left[E_{0}^{2}(t)-E_{0}^{2}(0)\right]
$$

With the aid of this relation, the average of $\Delta \omega=\Delta \omega_{0}-\left(k^{2} c^{2} / \omega-\omega_{0}\right)\left(\gamma_{1}-\gamma_{10}\right) / \gamma_{1}$ becomes

$$
\begin{aligned}
\langle\Delta \omega\rangle= & \left\langle\Delta \omega_{0}\right\rangle+\left(\omega_{p b}^{2} / \Omega_{0}\right)\left[\varepsilon_{0}\left(1+\varepsilon_{r}\right) / 2 \gamma_{0}\right. \\
& \left.\Delta n_{\varepsilon} m c^{2}\right]\left[E_{0}^{2}(t)-E_{0}^{2}(0)\right]
\end{aligned}
$$

where the dispersion relation $\varepsilon_{r}=(k c / \omega)^{2}$ has been used; $\left\langle\Delta \omega_{0}\right\rangle=1 / 2 \quad\left(\left\langle\Delta \omega_{01}\right\rangle-\left\langle\Delta \omega_{02}\right\rangle\right) \sim\left\langle\Delta \omega_{01}\right\rangle$. Since $\left\langle\Delta \omega_{0}\right\rangle<0$,

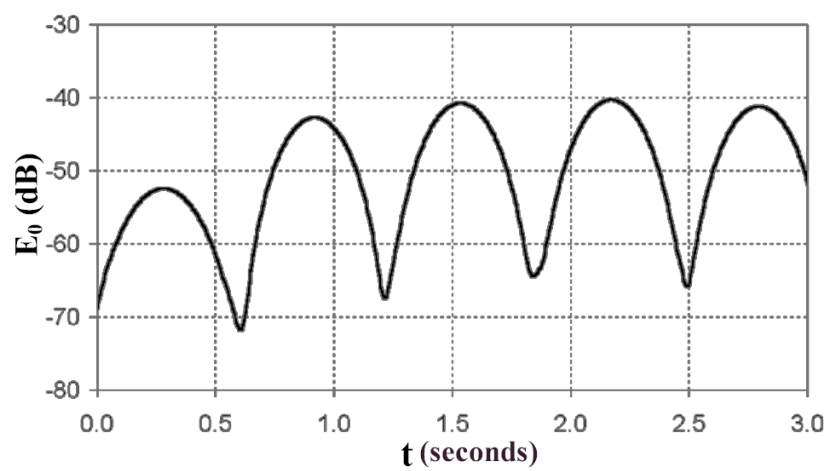

Fig. 1. Temporal evolution of the amplitude of a whistler wave propagating in the magnetosphere.

Eq. (7) indicates that the mismatch frequency $|\langle\Delta \omega\rangle|$ decreases as the wave amplitude $E_{0}$ increases, a positive feedback for energy transfer from resonant electrons to the wave.

The governing Eq. (C11) for the self-consistent field amplitude $E_{0}(\mathrm{t})$ is now analyzed numerically. We first normalize Eq. (C11) to a dimensionless form by introducing $X=\left[\varepsilon_{0}\left(\varepsilon_{r}-1\right)\left|\left\langle\Delta \omega_{0}\right\rangle\right| \quad \mid 4 \omega \gamma_{10} \Delta n_{\varepsilon} m c^{2}\right]^{1 / 2} E_{0}(t)$, $\xi=\left|\left\langle\Delta \omega_{0}\right\rangle\right| t, \quad$ and $\quad g=\left(n_{\varepsilon} / \Delta n_{\varepsilon}\right)\left(\varepsilon_{r}+1\right) \omega \omega_{p b}^{2} / \Omega_{0}\left(\varepsilon_{r}-1\right)$ $\left\langle\Delta \omega_{0}\right\rangle^{2}=\left(\mathrm{n}_{\varepsilon} / \Delta n_{\varepsilon}\right)\left(\varepsilon_{r}+1\right)\left(\omega /\left\langle\Delta \omega_{0}\right\rangle\right)^{2} . \quad$ The normalized equation has the form

$\left[d_{\xi}^{3}-48 X f_{c} d_{\xi}^{2}+\left(1+b X^{2}+48 f_{s} X\right) d_{\xi}\right] X$

$=16\left[1-g\left(X^{2}-X_{0}^{2}\right)\right] f_{c}$

where $X_{0}=X(0) ; f_{c}=\int_{0}^{\xi} X\left(\xi^{\prime}\right) \cos \varphi\left(\xi, \xi^{\prime}\right) d \xi^{\prime}$ and $f_{s}=-\int_{0}^{\xi} X\left(\xi^{\prime}\right) \sin \varphi(\xi, \xi) d \xi^{\prime} ; \varphi\left(\xi, \xi^{\prime}\right)=\int_{\xi^{\prime}}^{\xi}$ $\left\{1-2 g\left[X^{2}\left(\xi^{\prime \prime}\right)-X_{0}^{2}\right]\right\} d \xi^{\prime \prime} ; b=\left[4 \Delta \omega_{p \varepsilon}^{2} k^{2} c^{2} / \gamma_{10}\left(\varepsilon_{r}-1\right)\right.$ $\left.\left|\left\langle\Delta \omega_{0}\right\rangle\right|^{3} \omega\right]\left[\left(1+v_{z 0} \omega / k c^{2}\right)+3\left(\omega \Omega_{0} / \gamma_{10} k^{2} c^{2}\right)-(9 / 4) \alpha_{0}^{2}\right.$ $\left.\left(\omega \Omega_{0} / \gamma_{10} k^{2} c^{2}\right)^{2}\right] \cong 192\left[\left(\varepsilon_{r}+1\right) /\left(\varepsilon_{r}-1\right)\right]\left(\omega \gamma_{10} / \alpha_{0}^{2} \Omega_{0}\right)$; $b$ and $g$ are constant coefficients; Eq. (8) is subjected to the initial conditions: $X(0)=X_{0}, d_{\xi} X(0)=\sqrt{ } 3 X_{0}, d_{\xi}^{2} X(0)=3 X_{0}$.

The background parameters give $b=1440$ and $g=2 \times 10^{7} \quad$ (i.e., $\quad \varepsilon_{r} \sim 4$ and $\left|\left\langle\Delta \omega_{0}\right\rangle\right| \mid \omega \sim 5 \times 10^{-4}$ ); setting $X_{0}=3.58 \times 10^{-4}$, Eq. (8) is solved by an ODE solver. The result is presented in Fig. 1, showing the temporal evolution of the field amplitude $E_{0}(t)$. The dB-scale plot in Fig. 1 is for a direct comparison with the early Siple experimental result (Helliwell et al., 1980; Helliwell and Inan, 1982; Helliwell, 1983). In Siple experiments (Helliwell et al., 1980), injected Siple signals of $3 \mathrm{kHz}$, propagating along the $L \cong 4$ shell, were often amplified by 10 to $30 \mathrm{~dB}$ and oscillated in time. The numerical result presented in Fig. 1 also indicates that whistler wave amplitude can indeed be amplified more than $25 \mathrm{~dB}$ by $\mathrm{keV}$ electrons through loss-cone negative mass instability, and also oscillates in time in a similar fashion. A good agreement between the numerical and experimental results is obtained. It is worth to point out that the wave amplitude oscillation feature observed in Siple experiments 


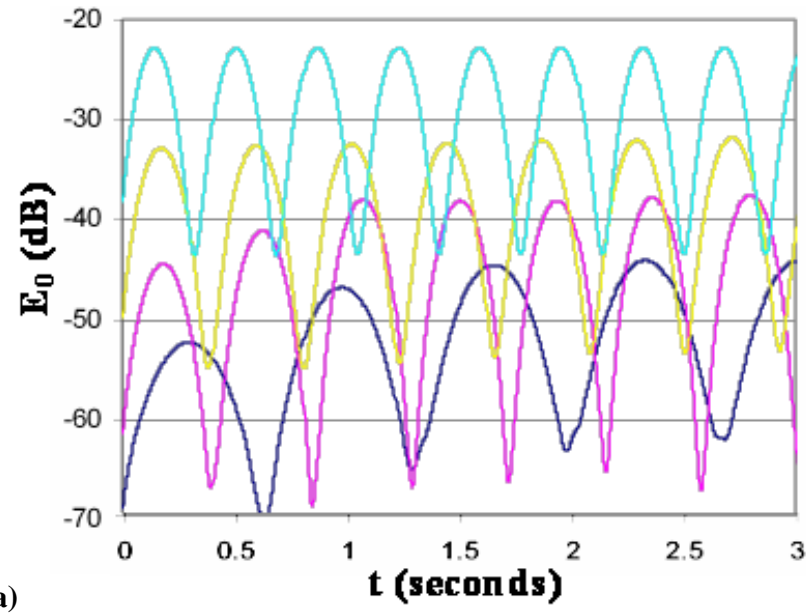

(a)

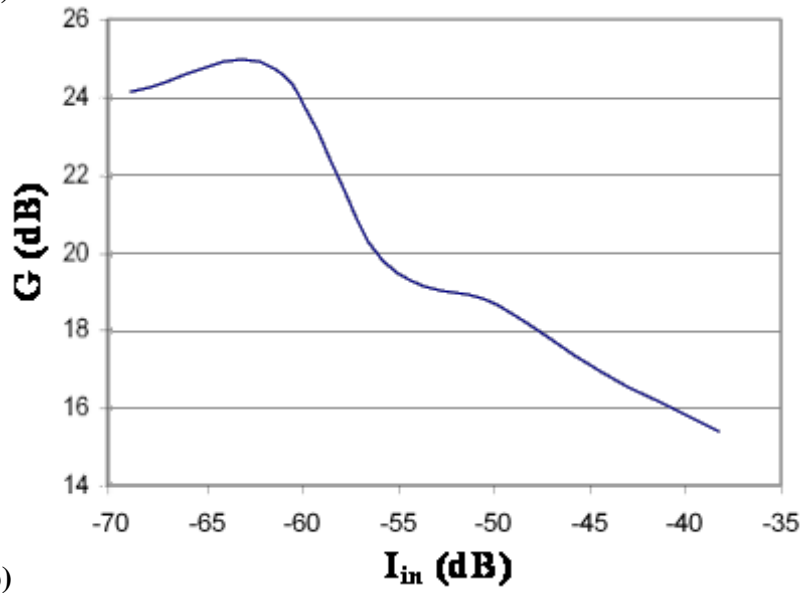

Fig. 2. (a) Wave amplitude verses time for four different incident wave intensities and (b) amplitude gain $G$ of amplified whistler wave verses the incident wave intensity.

is different from the pattern of pulsations appearing in the natural event of chorus, which has been simulated by the quasi-periodic ELF/VLF generation model (Pasmanik et al., 2004).

The dependence of the wave amplitude gain $G=20 \mathrm{log}$ $\left(E_{0 m} / E_{i n}\right)$ on the density of energetic electrons is through the parameter $g$ in Eq. (8), where $E_{0 m}$ and $E_{i n}$ are the maximum amplitude of the amplified wave and the amplitude of the incident wave; $g \propto\left(n_{\varepsilon} / \Delta n_{\varepsilon}\right)\left(\omega /\left\langle\Delta \omega_{0}\right\rangle\right)^{2} \propto n_{\varepsilon} / \Delta n_{\varepsilon}^{5 / 3} \propto n_{\varepsilon}^{-2 / 3}$. The numerical analysis shows that $\left(E_{0 m} / E_{i n}\right)^{2} \propto n_{\varepsilon}$, i.e., the gain $G$ increases linearly with the logarithm of the density of energetic electrons.

It is noted that the field amplitude $E_{i n}$ of the incident whistler wave in Fig. 1 is rather low (to be consistent with that of previous experiments (Helliwell et al., 1980)). In practical application for achieving significant electron precipitation, the incident wave field has to increase considerably. Thus, it is important to realize how the gain $G$ varies with the incident wave intensity for a fixed background con- dition, where the wave frequency is in the range to achieve double cyclotron resonances. This is exemplified by considering a case with the following background conditions: $\Omega_{0} / \omega=8, \gamma_{10}=1.1$, and $\varepsilon_{r}=41 ;\left|\left\langle\Delta \omega_{0}\right\rangle\right| / \omega \sim 1.287 \times 10^{-3}$ and $v / c=0.417$; setting $v_{\perp} / c=0.32$, leads to $\alpha_{0}=0.173, b=428$, and $g=1.06 \times 10^{7}$. Presented in Fig. $2 \mathrm{a}$ are the plots of wave intensity verse time for four different intensity levels of the incident wave. As shown both the gain and the oscillating period of the wave intensity decrease as the initial wave intensity increases. The gain function $G\left(I_{i n}\right)$ is plotted in Fig. $2 \mathrm{~b}$, where $I_{i n}$ is proportional to $E_{i n}^{2}$. It shows that with a $30 \mathrm{~dB}$ increase of $I_{i n}, G$ reduces only about $10 \mathrm{~dB}$; in other words, the amplification process remains effective for a large increase of $I_{i n}$.

\section{Formulation and analysis of chaotic scattering process}

Interaction between trapped energetic electrons (hundreds of $\mathrm{keV}$ to $\mathrm{MeV}$ ) and a large amplitude whistler wave is considered. These electrons are trapped by the magnetic mirror of the geomagnetic field and bounce back and forth about the geomagnetic equator. Since these electrons are practically collisionless, single particle approach will be adopted. To simplify the formulation while retaining the essential physics, the magnetic dipole field is modeled by a parabolic scalar potential, $\varphi=-m \omega_{b}^{2} z^{2} / 2 e$, superimposed over a uniform magnetic field, $\mathbf{B}_{0}=B_{0} \hat{z}$, where $z$ is the distance from the equatorial plane. This parabolic potential characterized by a bounce frequency $\omega_{b}$ simulates the mirror effect of the magnetic dipole field (Ho et al., 1994). Justification for this simplification of the background magnetic field configuration is given in Appendix D.

\subsection{Formulation}

The total vector potential in the system, contributed by both the wave and static fields, is given by $\mathbf{A}=\mathbf{A}_{w}+\hat{\mathbf{y}} B_{0} x$, where $\mathbf{A}_{w}$, the vector potential of the whistler wave fields, is expressed explicitly to be

$\mathbf{A}_{w}=(B / k)[\hat{\boldsymbol{x}} \cos (k z-\omega t)+\hat{\boldsymbol{y}} \sin (k z-\omega t)]$

With both of the potentials given and let $p$ be the canonical momentum, the electron relativistic Hamiltonian $H(\mathbf{r}, \mathbf{p})$, is given by

$H=c\left[(\mathbf{p}+e \mathbf{A})^{2}+m^{2} c^{2}\right]^{1 / 2}-e \varphi$

This Hamiltonian yields trajectories in a six-dimensional phase space. However, it can be simplified to reduce the degree of freedom. The y-coordinate is cyclic, $p_{y}=$ constant, which can be set equal to zero without losing the generality. We next transform the canonical coordinates $\left(x, p_{x}\right)$ to a new 
pair $(Q, P)$ by introducing the generating function (Faith et al., 1997a, b)

$F_{1}(x, Q, t)=1 / 2 m \Omega_{0}\left(x^{2}+Q^{2}\right) \cot \omega t-m \Omega_{0} x Q \csc \omega t(11)$

Equation (11) is applied for the canonical transformation: $p_{x}=\partial F_{1} / \partial x$ and $P=-\partial F_{1} / \partial Q$, to determine the new coordinates

$$
\begin{aligned}
& P=p_{x} \cos \omega t+m \Omega_{0} x \sin \omega t, \\
& Q=-\left(p_{x} / m \Omega_{0}\right) \sin \omega t+x \cos \omega t
\end{aligned}
$$

and the new Hamiltonian

$$
\begin{aligned}
K= & H+\partial F_{1} / \partial t= \\
& c\left\{P^{2}+p_{z}^{2}+\left(m \Omega_{0} Q\right)^{2}+m^{2} c^{2}+\left(m \Omega_{1} / k\right)^{2}\right. \\
& \left.+2 m\left(\Omega_{1} / k\right)\left[P \cos k z-m \Omega_{0} Q \sin k z\right]\right\}^{1 / 2} \\
& +1 / 2 m \omega_{b}^{2} z^{2}-1 / 2 \omega\left(m \Omega_{0} Q^{2}+P^{2} / m \Omega\right)
\end{aligned}
$$

where $\Omega_{1}=e B / m$. From Eq. (13), the Hamilton's equations of motion, $d \mathbf{r} / d t=\nabla_{p} K$ and $d \mathbf{p} / d t=-\nabla K$, can be derived. Use of the normalizations: $k^{2} K / m \omega^{2}=K^{\prime} \rightarrow K$, $k Q=Q^{\prime} \rightarrow Q, k P / m \omega=P^{\prime} \rightarrow P, k z=z^{\prime} \rightarrow z$, $k p_{z} / m \omega=p_{z}^{\prime} \rightarrow p_{z}, \Omega_{0} / \omega=\Omega_{0}^{\prime} \rightarrow \Omega_{0}, \Omega_{1} / \omega=\Omega_{1}^{\prime} \rightarrow \Omega_{1}$, $\omega t=t^{\prime} \rightarrow t$, and $\omega_{b} / \omega=\omega_{b}^{\prime} \rightarrow \omega_{b}$, we arrive at the normalized relativistic equations of motion convenient for later numerical analysis:

$d P / d t=-\Omega_{0}\left(\Omega_{0} / \gamma_{2}-1\right) Q+\left(\Omega_{0} \Omega_{1} / \gamma_{2}\right) \sin z$

$d Q / d t=\left(\Omega_{0} / \gamma_{2}-1\right)\left(P / \Omega_{0}\right)+\left(\Omega_{1} / \gamma_{2}\right) \cos z$

$d z / d t=p_{z} / \gamma_{2}$

$d p_{z} / d t=-\omega_{b}^{2} z+\left(\Omega_{1} / \gamma_{2}\right)\left(P \sin z+\Omega_{0} Q \cos z\right)$

where $\gamma_{2}=(\omega / k c)\left[(k c / \omega)^{2}+P^{2}+p_{z}^{2}+\Omega_{0}^{2} Q^{2}+\Omega_{1}^{2}+2 \Omega_{1}(P \cos z-\right.$ $\left.\left.\Omega_{0} Q \sin z\right)\right]^{1 / 2}$, is the relativistic factor; $\omega / k c$, the normalized phase velocity of whistler wave, will be taken to be $1 / 2$ in the numerical analysis. This value of the phase velocity corresponds quite well to the case of the magnetosphere.

Since $K$, as given by Eq. (13), does not depend on $t$ explicitly, it is a constant of motion, i.e., $d K / d t=\partial K / \partial t=0$, which reduces the degree of freedom of the system by one. Thus the set of Eqs. (14-17) describes trajectories in a threedimensional space. A surface of section technique is used to further reduce the three-dimensional continuous time system to a two-dimensional map, where we can examine the chaoticity of the system graphically.

\subsection{Numerical analysis}

The constant of the motion, $K=$ constant, allows us to eliminate one of the four variables in Eqs. (14-17). In the following analysis, we choose to eliminate the variable $P$, i.e., Eq. (14). We also choose $Q=0$ as the surface of section to

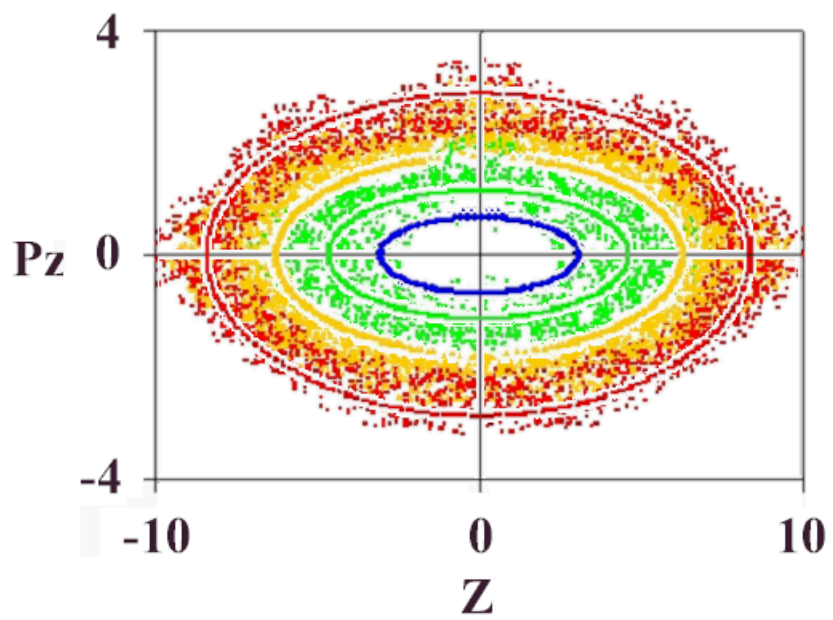

Fig. 3. Surface of section plot from the trajectories of four electrons with initial energies $\gamma_{0}=1.2$ (in blue), 1.5 (in green), 2 (in orange), and 3 (in red), and with the same initial pitch angle $\theta_{0}=60^{\circ}$ and wave amplitude $\Omega_{1}=0.08$ in a system with $\Omega_{0}=8$ and $\omega_{b}=0.1$. The unperturbed trajectories $\left(\Omega_{1}=0\right)$ represented by elliptical curves are superimposed in the same plot.

project trajectories onto the $z-p_{z}$ plane or graphically depicting the chaoticity of the system. It is noted that for a given $K$, both $P$ and $d Q / d t$ are double valued. Hence, the surface of section is separated into $d Q / d t<0$ and $d Q / d t>0$ cases. We choose only to present the $d Q / d t<0$ case because the results of the two cases are mirror images of each other. Equations (15-17) are integrated in time using a fifth order Runge Kutta ODE solver. When the sign of $Q$ changes from positive to negative between two time steps, the trajectory between these two points is interpolated to the $Q=0$ plane, and the resulting point recorded. The time integration continues for about 1000 bounce periods. The initial conditions $\left(Q_{0}, z_{0}, P_{0}, p_{z 0}\right)$ of Eqs. (15-17) are determined as follows. Consider an electron having an initial energy $\gamma_{20}$ and pitch angle $\theta_{20}$ at equator, where $\theta_{20}=\tan ^{-1}\left(P_{0} / p_{z 0}\right)$, and set $Q_{0}=0=z_{0}$, hence, $\gamma_{20}=1 / 2\left(4+P_{0}^{2}+p_{z 0}^{2}\right)^{1 / 2}$. We can then obtain the remaining two initial conditions $P_{0}=2\left(\gamma_{20}^{2}-\right.$ $1)^{1 / 2} \sin \theta_{20}$ and $p_{z o}=2\left(\gamma_{20}^{2}-1\right)^{1 / 2} \cos \theta_{20}$. The trajectories of different electrons represented by different initial energies $\gamma_{20}$ are examined by the surface of section technique. As these electrons interact with the wave having normalized amplitude $\Omega_{1}$, the behavior of each trajectory, which is regular or chaotic, is expected to depend strongly on two quantities, the value of $\gamma_{20}$ and the wave amplitude represented by $\Omega_{1}$.

We now use surface of section approach to characterize the interaction of a $3 \mathrm{kHz}$ whistler wave with energetic electrons in $L=3.3$ shell. The parameters of the system are normalized to be $\Omega_{0}=8$ and $\omega_{b}=0.1$. The dependence of the chaoticity of the system on the electron energy, i.e., $\gamma_{20}$, is examined by mapping the trajectories of four electrons having $\gamma_{20}=1.2,1.5,2$, and 3 , in the same surface of section 


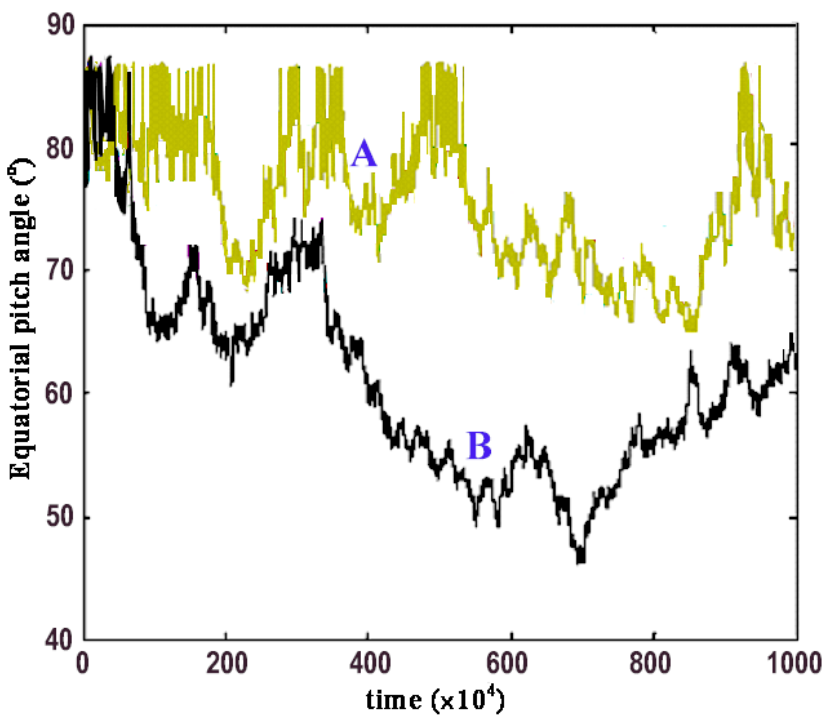

Fig. 4. Temporal evolution of the pitch angle of a $1.5 \mathrm{MeV}$ electron interacting with a whistler wave at Doppler shifted cyclotron resonance. Plots $\mathrm{A}$ and $\mathrm{B}$ correspond to wave magnetic field $B_{1}=7 \times 10^{-4} B_{0}$ and $8.1 \times 10^{-4} B_{0}$ cases, respectively.

plot. Presented in Fig. 3 is the one corresponding to $\Omega_{1}=0.08$ ( $1 \%$ of the background magnetic field) and the same initial pitch angle $\theta_{20}=60^{\circ}$. The elliptical curves in Fig. 3 represent the unperturbed trajectories $\left(\Omega_{1}=0\right.$ case), which are superimposed in the plot for a comparison. As shown, the trajectories for $\gamma_{20}=1.5,2$, and 3 are mapped in a large phase space region, suggesting the occurrence of significant chaotic pitch angle scattering. Indeed, it is found that the electron equatorial pitch angle $\theta$, in the three chaotic cases, can become as low as that less than $30^{\circ}$. However, the trajectory in $\gamma_{20}=1.2$ case remains regular, demonstrating that the electron energy is important to the type of motion the electron undergoes. The wave amplitude required causing an electron trajectory to become chaotic is lower for those electrons with energy $>100 \mathrm{keV}$. This confirms that one can use the lower energy $(<100 \mathrm{kev})$ electrons to amplify whistler waves, which become more effective in precipitating higher energy ( $>1 \mathrm{MeV})$ electrons.

\section{Double cyclotron resonances for effective precipita- tion of $\mathrm{MeV}$ electrons by whistler waves}

A wave resonant with $\mathrm{MeV}$ electrons $\left(\gamma=\gamma_{2}\right)$ satisfies a resonance condition, similar to that given by Eq. (1),

$\omega=\Omega_{0} / \gamma_{2}+k P_{z 2} / \gamma_{2} m$

We now find the initial conditions of electrons such that Eqs. (1) and (18) can be satisfied simultaneously. The relationships and notations to be applied are first introduced as follows: $\Omega_{0} \propto L^{-3} ; \omega_{p b} \propto L^{-3 / 2} ; \omega_{b} \propto\left(1-\gamma_{2}^{-2}\right)^{-1 / 2} \Omega_{0} / L$ is the bounce frequency of $\mathrm{MeV}$ electrons; $n \cong\left(\omega_{p b}^{2} / \omega \Omega_{0}\right)^{1 / 2}$ is the index of refraction of the background cold plasma on the whistler wave; $L$ is the number of earth radii, i.e., $L$ value of a magnetic flux tube; $\theta_{1}=\tan ^{-1}(j)^{1 / 2}$ is the initial pitch angle of $\mathrm{keV}$ electrons which contribute to wave amplification, thus $\cos \theta_{1}=1 /(1+j)^{1 / 2} ; \gamma_{1} \sim 1.1$ is the relativistic factor of $\sim 50 \mathrm{keV}$ electrons; $\theta_{2}$ is the initial pitch angle of $\mathrm{MeV}$ electrons which are being precipitated; the normalized phase velocity of the wave $V=v_{p} / c=\left(\Omega_{0} / \omega_{p}\right) \xi^{-1 / 2}$, where $\xi=\Omega_{0} / \omega$.

With the aid of some known background parameters: $n_{b} \sim 280 \mathrm{~cm}^{-3}$ at $L=4.9, \quad B_{0} \sim 0.25$ Gauss at $L=1$, and $\omega_{b} / \Omega_{0} \sim(1 / 3) \times 10^{-2}$ for $\gamma_{2}=3$ and $L=2$, we can obtain the functional dependence of the background parameters on $L$ as $\omega_{p b}=2 \pi \times 1.63 \times 10^{6} / L^{3 / 2}, \Omega_{0}=2 \pi \times 7 \times 10^{5} / L^{3}$, and $\omega_{b} / \Omega_{0}=\left(\omega_{b} / \omega\right) /\left(\Omega_{0} / \omega\right)=7.07 \times 10^{-3}\left(1-\gamma_{2}^{-2}\right)^{-1 / 2} / L$. The resonance conditions (1) and (18) and the dispersion relation lead to

$\Omega_{0} / \omega=\gamma_{2}+n\left(\gamma_{2}^{2}-1\right)^{1 / 2} \cos \theta_{2}=\xi=\gamma_{1}+n\left(\gamma_{1}^{2}-1\right)^{1 / 2} \cos \theta_{1}$

$n=2.33 L^{3 / 2} \xi^{1 / 2}=2.33 L^{3 / 2}\left[\gamma_{2}+n\left(\gamma_{2}^{2}-1\right)^{1 / 2} \cos \theta_{2}\right]^{1 / 2}$

Equation (19) leads to $n=\left(\gamma_{2}-\gamma_{1}\right) /\left[\left(\gamma_{1}^{2}-1\right)^{1 / 2} \cos \theta_{1}-\left(\gamma_{2}^{2}-\right.\right.$ $\left.1)^{1 / 2} \cos \theta_{2}\right]$, which is then used to re-express Eq. (19) as

$$
\begin{aligned}
\Omega_{0} / \omega \rightarrow \Omega_{0}= & {\left[\gamma_{2}\left(\gamma_{1}^{2}-1\right)^{1 / 2} \cos \theta_{1}-\gamma_{1}\left(\gamma_{2}^{2}-1\right)^{1 / 2}\right.} \\
& \left.\cos \theta_{2}\right] /\left[\left(\gamma_{1}^{2}-1\right)^{1 / 2} \cos \theta_{1}-\left(\gamma_{2}^{2}-1\right)^{1 / 2} \cos \theta_{2}\right]
\end{aligned}
$$

Equations (19) and (20) are solved to yield

$$
\begin{aligned}
\cos \theta_{2}= & 1 / 2\left[\left(\gamma_{1}^{2}-1\right) /\left(\gamma_{2}^{2}-1\right)\right]^{1 / 2} \\
& \times\left\{\left(\gamma_{2} / \gamma_{1}+1\right) \cos \theta_{1}-\left(\gamma_{2} / \gamma_{1}-1\right)\left[\cos ^{2} \theta_{1}\right.\right. \\
& \left.\left.+4 \gamma_{1} / 5.43 L^{3}\left(\gamma_{1}^{2}-1\right)\right]^{1 / 2}\right\}
\end{aligned}
$$

The normalized (to $\omega$ ) bounce frequency of electrons and normalized (to $c$ ) phase velocity of the wave are given by $\omega_{b}=7.07 \times 10^{-3} \quad\left(1-\gamma_{2}^{-2}\right)^{-1 / 2} \Omega_{0} / L$ and $V=0.432 L^{-3 / 2} \Omega_{0}^{-1 / 2}$

We now consider the $\gamma_{2}=4$ case that precipitates $1.5 \mathrm{MeV}$ electrons. From Eq. (21), $\theta_{2}=86.5^{\circ}$. We then have $\Omega_{0}=8.6038, \quad V=0.0517$, and $\omega_{b}=2.945 \times 10^{-2}$. Equations (15) to (17) are now integrated numerically to evaluate the pitch angle scattering, resulting from the wave-electron resonance interaction. Presented in Fig. 4 is a result that double resonance condition is satisfied.

As shown, with $\Omega_{1}=6 \times 10^{-3}$ (i.e., the wave magnetic field $\left.B_{1}=7 \times 10^{-4} B_{0}\right)$, the electron trajectory is chaotic but the pitch angle (plot $A$ ) of the scattered electron remains larger than $60^{\circ}$. However, as the wave magnetic field $B_{1}$ increases slightly to $8.1 \times 10^{-4} B_{0}$ (i.e., $\Omega_{1}=7 \times 10^{-3}$ ), significantly large pitch angle scattering occurs. As shown, electron is scattered to a pitch angle $<50^{\circ}$ (plot B), less than the loss cone angle. The required wave magnetic field amplitude is calculated to be about $0.08 \%$ (i.e., $\left.\Omega_{1} / \Omega_{0}=0.007 / 8.6038=0.0008\right)$ of the background magnetic filed. This is an example that a whistler wave, with proper 
parameters, can be amplified by the $\mathrm{keV}$ electrons and simultaneously scatters $\mathrm{MeV}$ electrons, both processes via the cyclotron resonance interaction. The results show that cyclotron resonant interaction reduces the required field amplitude, for achieving effective chaotic scattering, by a factor more than 20. On the other hand, the required interaction time is also increased by an order of magnitude.

\section{Summary}

Small pitch angle scatterings in cyclotron resonance interaction with a whistler wave can diffuse $\mathrm{MeV}$ electrons, with pitch angles close to the loss cone angle, into loss cones (Albert, 2000). On the other hand, it will need a chaotic scattering process to precipitate those deeply trapped electrons; the wave field has to exceed a threshold (Kuo et al., 2004).

Amplification of whistler wave by (tens of $\mathrm{keV}$ ) energetic electrons in the magnetosphere through loss-cone negative mass instability is studied. The theory is formulated and the numerical result is shown to agree well with the experimental result, both qualitatively and quantitatively. Such amplification reduces considerably the required field intensity of the incident whistler wave for the purpose of precipitating $\mathrm{MeV}$ electrons in the magnetosphere.

The feasibility to invoke a double cyclotron resonance situation for an optimal approach to reduce the population of $\mathrm{MeV}$ electrons trapped in the magnetosphere is then demonstrated. In this approach, the wave is first amplified by (tens of $\mathrm{keV}$ ) energetic electrons; once the wave field exceeds the threshold for the commencement of chaos, cyclotron resonance-enhanced chaotic scattering can precipitate deeply trapped $\mathrm{MeV}$ electrons into loss cones. The numerical results demonstrate that a $1.5 \mathrm{MeV}$ electron can be scattered from an initial pitch angle of $86.5^{\circ}$ to a pitch angle $<50^{\circ}$ by a whistler wave with the magnetic field amplitude of $0.08 \%$ of the background magnetic field, which is about 20 times smaller than that without invoking cyclotron resonance. This percentage converts to about $3100 \mathrm{pT}$ at $L=2$, and $200 \mathrm{pT}$ at $L=5$, which reduce to $550 \mathrm{pT}$ and $36 \mathrm{pT}$, respectively, after taking advantage of the $15 \mathrm{~dB}$ gain.

Finally, it should be pointed out that this optimal approach, relying on electron cyclotron resonance interaction, requires that the wave have a broad frequency spectrum, so that a considerable fraction of the very energetic electrons can be precipitated simultaneously.

\section{Appendix A}

\section{Resonant trajectory equations}

The equations for the electron motion in a dc magnetic field $\hat{z} B_{0}$ and right-hand circularly polarized wave fields $\mathbf{E}$ and $\mathbf{B}$ are given by

$d \boldsymbol{r} / d t=\mathbf{p} / \gamma_{1} m$

$d \boldsymbol{p} / d t=-e\left[\mathbf{E}+\boldsymbol{v} \times\left(\mathbf{B}+\hat{z} B_{0}\right)\right]$

$m c^{2} d \gamma_{1} / d t=e \mathbf{E} \cdot \mathbf{v}$

where $\quad \gamma_{1}=\left(1+p^{2} / m^{2} c^{2}\right)^{1 / 2}, \quad \boldsymbol{p}=\gamma_{1} m \boldsymbol{v}, \quad$ and the whistler wave fields $\mathbf{E}$ and $\mathbf{B}$ are $\mathbf{E}=E_{0}(t) \hat{\boldsymbol{u}}$ and $\mathbf{B}=(k / \omega) E_{0}(t) \hat{\boldsymbol{v}} ; \quad \hat{\boldsymbol{u}}=[\hat{\boldsymbol{x}} \cos (k z-\omega t)-\hat{\boldsymbol{y}} \sin (k z-\omega t)] \quad$ and $\hat{\boldsymbol{v}}=[\hat{\boldsymbol{x}} \sin (k z-\omega t)+\hat{\boldsymbol{y}} \cos (k z-\omega t)] ; \omega$ and $k$ are related by the whistler wave dispersion relation $\omega=\Omega_{0} k^{2} c^{2} / \omega_{p b}^{2}$ given in Eq. (2); $\varepsilon_{0}$ is the free-space permitivity.

When the wave frequency $\omega$ is near the Doppler shifted electron cyclotron frequency, $\omega \cong \Omega_{0} / \gamma_{1}-k v_{z}$, the electron trajectory is mainly governed by the cyclotron resonance interaction. After removing all the fast oscillating components in the Lorentz force, Eqs. (A1-A3) reduce to a set of selfconsistent governing equations for the slowly time varying functions $\gamma_{1}, v_{\perp}, v_{z}$ and $\Phi$ (Kuo and Cheo, 1985)

$$
\begin{aligned}
& d \alpha / d t=-\left(k / \Omega_{0}\right)\left(e E_{0} / m\right)\left(1+k v_{z} / \omega\right) \cos \phi \\
& d \phi / d t=-\Delta \omega+\left(k / \Omega_{0}\right)\left(e E_{0} / m\right)\left(1+k v_{z} / \omega\right) \alpha^{-1} \sin \phi \\
& d v_{z} / d t=\left(\Omega_{0} / k\right)\left(e E_{0} / m c^{2}\right)\left(\alpha / \gamma_{1}^{2}\right)\left(k c^{2} / \omega+v_{z}\right) \cos \phi \\
& d \gamma_{1} / d t=-\left(\Omega_{0} / k\right)\left(e E_{0} / m c^{2}\right)\left(\alpha / \gamma_{1}\right) \cos \phi
\end{aligned}
$$

where $\quad \alpha=k v_{\perp} / \Omega_{0} \quad$ and $\quad \phi=k z_{0}+\left[\Theta_{0}+\Phi(t)\right]-\int_{0}^{t} \Delta \omega^{\prime} d t^{\prime}$; $\Delta \omega=\Delta \omega_{0}+\Omega_{0}\left(\gamma_{1}-\gamma_{10}\right) / \gamma_{1} \gamma_{10}+k\left(v_{z}-v_{z 0}\right), \quad \Delta \omega_{0}=\omega-\omega_{0}$, and $\omega_{0}=\Omega_{0} / \gamma_{10}-k v_{z 0} ; \quad \Theta_{0}=\tan ^{-1}\left(v_{y 0} / v_{x 0}\right), \Phi$ accounts for the phase shift in the electron gyration due to interaction with the wave fields, $\gamma_{10}$ is the initial relativistic factor of the resonant electrons. The ratio of Eqs. (A6) and (A7) leads to an invariant relation $\gamma_{1}\left(k c^{2} / \omega+v_{z}\right)=$ const. $=\gamma_{10}\left(k c^{2} / \omega+v_{z 0}\right)$, which is used to obtain $\Delta \omega=\Delta \omega_{0}-\left(k^{2} c^{2} / \omega-\omega_{0}\right)\left(\gamma_{1}-\gamma_{10}\right) / \gamma_{1}$. It is noted that $k^{2} c^{2} / \omega-\omega_{0}>0$ for whistler waves considered in the present case, i.e., $\Delta \omega$ increases as electron loses energy to the wave and vice versa.

\section{Appendix B}

\section{Collective effect}

The $\mathrm{keV}$ electron plasma has a loss-cone distribution given by Eq. (3) and its distribution in $P_{z}$ is yet a Maxwellian given by Eq. (4) and shown in Fig. B1. Only a fraction of total electrons, e.g., in the two shade regions in Fig. B1, are close to Doppler shifted cyclotron resonance with the wave. In fact, the wave is experiencing cyclotron damping to the electrons which are initially at exact cyclotron resonance with the wave, i.e., $\Delta \omega_{0}=0$. On the other hand, the wave can exchange energy with other electrons having a mismatch frequency 


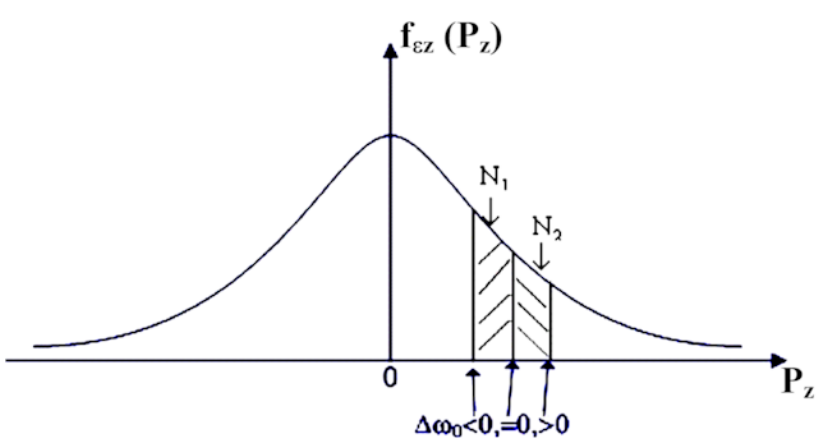

Fig. B1. Momentum distribution of energetic electrons and the regions close to cyclotron resonant interaction with the wave.

$\Delta \omega_{0}$ slightly different from zero. Depending on the initial phases of those electrons, the interaction can cause them either to gain energy from, or lose energy to, the wave, initially. In region $1, \Delta \omega_{01}<0$, one half of the electrons will lose energy to the wave initially, those electrons also reduce the mismatch frequency in the interaction (because $\gamma_{1}-\gamma_{10}<0$ ); it results in the increase of the interaction period of losing energy to the wave. The other half of the electrons, which gain energy from the wave initially, will increase their mismatch frequencies (because $\gamma_{1}-\gamma_{10}>0$ ) and reduce the interaction period of gaining energy from the wave. Therefore, those electrons with $\Delta \omega_{01}<0$ will lose energy to the wave on average.

For those electrons in region 2 with $\Delta \omega_{0}=\Delta \omega_{02}>0$, the above dynamic interaction process is reversed; on average, those electrons will gain energy from the wave. Using the definition $\Delta \omega_{0}=\omega-\omega_{0}=\omega-\Omega_{0} / \gamma_{10}+k v_{z 0}, \Delta \omega_{01}<\Delta \omega_{02}$ leads to $v_{z 01}<v_{z 02}$, which indicates that there are more electrons in the $\Delta \omega_{01}<0$ region than in the $\Delta \omega_{02}>0$ region (i.e., $N_{1}>N_{2}$ as shown in Fig. B1). Overall, the wave will gain energy from electrons and be amplified.

\section{Appendix C}

\section{Phase average relations and the governing equation of the wave amplitude}

The wave equation governs the self-consistent wave field

$\left[c^{2} \partial^{2} / \partial z^{2}-\partial^{2} / \partial t^{2}\right] \mathbf{E}=\varepsilon_{0}^{-1} \partial\left(\mathbf{J}_{e}+\mathbf{J}_{p}\right) / \partial t$

where $\mathbf{J}_{e}=-e n_{b} \boldsymbol{v}_{e}$ is the linear current density induced by the wave fields in the background plasma with $\boldsymbol{v}_{e}=-\left(e E_{0} / m \Omega_{0}\right) \hat{\boldsymbol{v}}$ and $\mathbf{J}_{p}$, the induced polarization current density associated with the resonant electrons, is given by

$$
\begin{aligned}
\mathbf{J}_{p}= & -e\left[N_{1}\left\langle\left(\hat{\boldsymbol{x}} v_{x}+\hat{\boldsymbol{y}} v_{y}\right)\right\rangle_{\varepsilon 1}+e N_{2}\left\langle\left(\hat{\boldsymbol{x}} v_{x}+\hat{\boldsymbol{y}} v_{y}\right)\right\rangle_{\varepsilon 2}\right] \\
= & -e\left\{N_{1}\left\langle\left\{\hat{\boldsymbol{u}} v_{\perp} \cos \phi+\hat{\boldsymbol{v}} v_{\perp} \sin \phi\right\}\right\rangle_{\varepsilon 1}+N_{2}\left\langle\left\{\hat{\boldsymbol{u}} v_{\perp} \cos \phi\right.\right.\right. \\
& \left.\left.\left.+\hat{\boldsymbol{v}} v_{\perp} \sin \phi\right\}\right\rangle_{\varepsilon 2}\right\} \\
= & \hat{\boldsymbol{u}} J_{p c}+\hat{\boldsymbol{v}} J_{p s}
\end{aligned}
$$

where $\quad J_{p c}=-e \Delta n_{\varepsilon}\left(\Omega_{0} / k\right)\left\langle\left(\alpha / \gamma_{1}\right) \cos \phi\right\rangle_{\varepsilon 1} \cong-P_{1}\langle\cos \phi\rangle_{\varepsilon 1}$, and $\quad J_{p s}=-e 2 n_{\varepsilon}\left(\Omega_{0} / k\right)\left\langle\left(\alpha / \gamma_{1}\right) \sin \phi\right\rangle_{\varepsilon 1} \cong-P_{2}\langle\sin \phi\rangle_{\varepsilon 1}$; $n_{\varepsilon}=\left(N_{1}+N_{2}\right) / 2$ and $P_{2}=2 n_{\varepsilon} e\left(\Omega_{0} / k\right)\left(\alpha_{0} / \gamma_{10}\right)$;

$\langle\sin \phi\rangle_{\varepsilon 2} \cong\langle\sin \phi\rangle_{\varepsilon 1}$ is assumed.

Comparing Eq. (6) with the average of Eq. (A7), leads to $\langle(\alpha / \gamma) \cos \phi\rangle_{\varepsilon}=\left(k / \Delta n_{\varepsilon} e \Omega_{0}\right)\left[\varepsilon_{0}\left(1+\varepsilon_{r}\right)\right] d_{t} E_{0}$, which is approximated to be

$\langle\cos \phi\rangle_{\varepsilon} \cong\left[\varepsilon_{0}\left(1+\varepsilon_{r}\right) / P_{1}\right] d_{t} E_{0}$

where $P_{1}=\Delta n_{\varepsilon} e\left(\Omega_{0} / k\right)\left(\alpha_{0} / \gamma_{0}\right)$ and the notation $d_{t}=d / d t$ is used.

Substitute $\mathbf{E}=E_{0}(t) \hat{\boldsymbol{u}}$ in Eq. (C1) and with the aid of Eq. (C2) and the dispersion relation $\varepsilon_{r}=1+\omega_{p}^{2} / \omega \Omega=k^{2} c^{2} / \omega^{2}$, Eq. $(\mathrm{C} 1)$ is reduced to

$d_{t}^{2} E_{0} \cong-\varepsilon_{0}^{-1}\left(d_{t} J_{p c}-\omega J_{p s}\right)$

$\omega\left(1+\varepsilon_{r}\right) d_{t} E_{0} \cong-\varepsilon_{0}^{-1}\left(d_{t} J_{p s}+\omega J_{p c}\right)$

Using the relation $J_{p c}=-\varepsilon_{0}\left(1+\varepsilon_{r}\right) d_{t} E_{0}$, Eq. (C5) reduces to $d_{t} J_{p s} \cong 0$, i.e., $\left\langle\left(d_{t} \phi\right) \cos \phi\right\rangle_{\varepsilon 1} \cong 0$, which, with the aid of Eq. (A6), leads to the relation

$s E_{0}\langle\sin 2 \phi\rangle_{\varepsilon 1} \cong 2\left\langle\Delta \omega_{0}\right\rangle\langle\cos \phi\rangle_{\varepsilon 1}$

where $s=\left(k / \Omega_{0}\right)(e / m)\left(1+k v_{z 0} / \omega\right) \alpha_{0}^{-1}$, and Eq. (C4) reduces to $J_{p s}=-\left(\varepsilon_{0} \varepsilon_{r} / \omega\right) d_{t}^{2} E_{0}$, which leads to

$\langle\sin \phi\rangle_{\varepsilon 1}=\left(\varepsilon_{0} \varepsilon_{r} / \omega P_{2}\right) d_{t}^{2} E_{0}$

Furthermore, with the aid of Eqs. (C7), (A4) and (A5) can be combined to obtain

$s E_{0}\langle\cos 2 \phi\rangle_{\varepsilon 1} \cong \omega\left(P_{2} / P_{1}\right)\left(1+\varepsilon_{r}^{-1}\right)\langle\sin \phi\rangle_{\varepsilon 1}$

$J_{p c}$ is the source current density of the radiation; it is usually governed by a second order differential equation. Introducing the relation

$$
\begin{aligned}
d_{t}^{2}\left\langle\left(\alpha / \gamma_{1}\right) \cos \phi\right\rangle= & -\left\langle\left[\left(\alpha / \gamma_{1}\right) d_{t}^{2} \phi+2 d_{t}\left(\alpha / \gamma_{1}\right) d_{t} \phi\right] \sin \phi\right\rangle \\
& -\left\langle\left[\left(\alpha / \gamma_{1}\right)\left(d_{t} \phi\right)^{2}-d_{t}^{2}\left(\alpha / \gamma_{1}\right)\right] \cos \phi\right\rangle(\mathrm{C} 9)
\end{aligned}
$$

With the aid of Eqs. (A4-A7), the right hand side terms of Eq. (C9) can be expressed explicitly in terms of the function $E_{0}(t)$ and its integrals and derivatives as follows

$$
\begin{aligned}
& \left\langle\left(\alpha / \gamma_{1}\right)\left(d_{t}^{2} \phi\right) \sin \phi\right\rangle \cong\left(\alpha_{0} / \gamma_{10}\right)(s / 2)\left\{d_{t} E_{0}(1-\langle\cos 2 \phi\rangle)\right. \\
& \quad-\left(A_{0} E_{0} / 2\right)\left(I_{c}\langle\sin \phi\rangle+I_{s}\langle\cos \phi\rangle\right) \\
& \quad+s\left[1+\left(\alpha_{0}^{2} / 2\right)\left(1+v_{z 0} \omega / k c^{2}\right)\right] E_{0}^{2}\langle\cos \phi\rangle-\left[\left\langle\Delta \omega_{0}\right\rangle\right. \\
& \left.\left.\quad+\alpha_{0}^{2} \omega\left(1+k v_{z 0} / \omega\right)\right] E_{0}\langle\sin 2 \phi\rangle\right\} \\
& \left.\quad\left\langle 2\left[d_{t}\left(\alpha / \gamma_{1}\right)\right]\left(d_{t} \phi\right) \sin \phi\right)\right\rangle \cong\left(\alpha_{0} / 2 \gamma_{10}\right)\left(s-b_{1} \alpha_{0} / \gamma_{10}^{2}\right) \\
& \quad\left[2\left\langle\Delta \omega_{0}\right\rangle E 0\langle\sin 2 \phi\rangle+A_{0} E_{0}\left(I_{c}\langle\sin \phi\rangle\right.\right. \\
& \left.\left.\quad+I_{s}\langle\cos \phi\rangle\right)-s E_{0}^{2}\langle\cos \phi\rangle\right] \\
& \quad\left\langle\left(\alpha / \gamma_{1}\right)\left(d_{t} \phi\right)^{2} \cos \phi\right\rangle \cong\left(\alpha_{0} / \gamma_{10}\right)
\end{aligned}
$$




$$
\begin{aligned}
& \left\{\left[\left\langle\Delta \omega_{0}\right\rangle^{2}+s^{2} E_{0}^{2} / 4+\left(A_{0}^{2} / 4\right)\left(I_{c}^{2}+I_{s}^{2}\right)\right]\langle\cos \phi\rangle\right. \\
& +\left(A_{0} / 2\right)\left(\langle\Delta \omega\rangle+\left\langle\Delta \omega_{0}\right\rangle\right) I_{c}-\left(s A_{0} E_{0} / 2\right) \\
& \left(I_{c}\langle\sin \phi\rangle+I_{s}\langle\cos \phi\rangle\right)-s E_{0}\left\langle\Delta \omega_{0}\right\rangle\langle\sin 2 \phi\rangle \\
& \left.+\left\langle\Delta \omega_{0}\right\rangle A_{0}\left(I_{c}\langle\cos 2 \phi\rangle+I_{s}\langle\sin 2 \phi\rangle\right)\right\} \\
& \left\langle\left[d_{t}^{2}\left(\alpha / \gamma_{1}\right)\right] \cos \phi\right\rangle \cong-\left(\alpha_{0} / 2 \gamma_{10}\right)\left\{\left(s-b \alpha_{0} / \gamma_{10}^{2}\right)\right. \\
& {\left[d_{t} E_{0}(1+\langle\cos 2 \phi\rangle)+\left(A_{0} E_{0} / 2\right)\left(I_{c}\langle\sin \phi\rangle\right.\right.} \\
& \left.+I_{s}\langle\cos \phi\rangle\right)-1 / 2\left(s-9 b_{1} \alpha_{0} / \gamma_{10}^{2}\right) E_{0}^{2}\langle\cos \phi\rangle \\
& \left.+E_{0}\left\langle\Delta \omega_{0}\right\rangle\langle\sin 2 \phi\rangle\right]+\left(3 s^{2} \alpha_{0}^{2} / 2\right) \\
& \left.\left(1+v_{z 0} \omega / k c^{2}\right) E_{0}^{2}\langle\cos \phi\rangle\right\}
\end{aligned}
$$

\section{Appendix D}

\section{Modeling a magnetic dipole field:}

The geomagnetic field localized around the electron guiding center resembles a parabolic mirror field. This mirror field may be expressed in local cylindrical coordinates $(r, \theta, z)$ as

$\mathbf{B}_{g}=B_{0}\left[1+\left(z^{2}-r^{2} / 2\right) / L^{2}\right] \hat{z}-\left(B_{0} z r / L^{2}\right) \hat{\boldsymbol{r}}$

where $L$ is the scale length of the magnetic field and $\mathbf{B}_{g}$ satisfies both $\boldsymbol{\nabla} \cdot \boldsymbol{B}_{g}=0$ and $\boldsymbol{\nabla} \times \boldsymbol{B}_{g}=0$ as required. The zcomponent of the equation of motion of an electron gyrating about the $\mathrm{z}$-axis in this mirror magnetic field is given by

where $A_{0}=\left(\omega_{p b}^{2} / \Omega_{0}\right)\left(b_{1} \alpha_{0} / \gamma_{10}^{2}\right), b_{1}=\left(\Omega_{0} / k\right)$ $\left(e / m c^{2}\right) ; I_{c}=\int_{0}^{t} E_{0}\left(t^{\prime}\right) \cos \left\langle\Delta \phi\left(t-t^{\prime}\right)\right\rangle_{\varepsilon 1} d t^{\prime}$ and $I_{s}=\int_{0}^{t} E_{0}\left(t^{\prime}\right) \sin \left\langle\Delta \phi\left(t-t^{\prime}\right)\right\rangle_{\varepsilon 1} d t^{\prime} ;\left\langle\Delta \phi\left(t-t^{\prime}\right)\right\rangle_{\varepsilon 1}=$ $-\int_{t^{\prime}}^{t}\langle\Delta \omega(\tau)\rangle_{\varepsilon 1} d \tau=-\left\langle\Delta \omega_{0}\right\rangle \int_{t^{\prime}}^{t}\left\{1+\left(\omega_{p b}^{2} / \Omega_{0}\left\langle\Delta \omega_{0}\right\rangle\right)\right.$ $\left.\left[\varepsilon_{0}\left(1+\varepsilon_{r}\right) / 2 \gamma_{10} \Delta n_{\varepsilon} m c^{2}\right]\left[E_{0}^{2}(\tau)-E_{0}^{2}(0)\right]\right\} d \tau$; the dispersion relation $k^{2} c^{2} / \omega-\omega \cong \omega_{p b}^{2} / \Omega_{0}$ is used; the higher order terms $\langle\cos 3 \phi\rangle_{\varepsilon 1}$ and $\langle\sin 3 \phi\rangle_{\varepsilon 1}$ are neglected and the approximations $\left\langle\cos \left(\phi-\phi^{\prime}\right)\right\rangle_{\varepsilon 1} \cong \cos \left\langle\left(\phi-\phi^{\prime}\right)\right\rangle_{\varepsilon 1} \cong \cos \left\langle\Delta \phi\left(t-t^{\prime}\right)\right\rangle_{\varepsilon 1}$ and $\left\langle\sin \left(\phi-\phi^{\prime}\right)\right\rangle_{\varepsilon 1} \cong \sin \left\langle\left(\phi-\phi^{\prime}\right)\right\rangle_{\varepsilon 1} \cong \sin \left\langle\Delta \phi\left(t-t^{\prime}\right)\right\rangle_{\varepsilon 1}$ are used in the derivation.

With the aid of the phase average relations (C3) and (C6$\mathrm{C} 10)$, the source terms on the right hand side of Eq. (C1) can be expressed explicitly in terms of the self-consistent field amplitude $E_{0}(t)$ and its derivatives and integrations. In essence, this is a procedure to combine the three first order differential Eqs. (A4) to (A6) to the wave Eq. (C1). It leads to a differential-integral equation for $E_{0}$ (Kuo et al., 2004)

$$
\begin{aligned}
& {\left[d_{t}^{3}+2 \Gamma d_{t}^{2}+\left(\Delta \underline{\omega}^{2}+\underline{C}\right) d_{t}\right] E_{0}=} \\
& a_{0}\left\{1+\left(n_{\varepsilon} / \Delta n_{\varepsilon}\right)\left(\omega_{p b}^{2} / \Omega_{0}\left\langle\Delta \omega_{0}\right\rangle\right)\left[\varepsilon_{0}\left(1+\varepsilon_{r}\right) / 4 \gamma_{10} \Delta n_{\varepsilon} m c^{2}\right]\right. \\
& \left.\times\left[E_{0}^{2}(t)-E_{0}^{2}(0)\right]\right\} \int_{0}^{t} E_{0}\left(t^{\prime}\right) \cos \left\langle\Delta \phi\left(t-t^{\prime}\right)\right\rangle_{\varepsilon 1} d t^{\prime}
\end{aligned}
$$

where $d_{t}$ stands for $d / d t ; \Gamma \cong-\left[3 \varepsilon_{r} / 8\left(1+\varepsilon_{r}\right)\right]$

$\left(b_{1} \alpha_{0} / \gamma_{10}^{2}\right) \times\left(A_{0} E_{0} I_{c} / \omega\right)$ and $\Delta \underline{\omega}^{2}=\left\langle\Delta \omega_{0}\right\rangle^{2}+\alpha_{0}^{2}\left\{s^{2}\right.$ $\left[\left(1+v_{z 0} \omega / k c^{2}\right)+3(\omega / k c)^{2}\left(1+k v_{z 0} / \omega\right)-\right.$ $\left.\left.(9 / 4) \alpha_{0}^{2}(\omega / k c)^{4}\left(1+k v_{z 0} / \omega\right)^{2}\right] E_{0}^{2}-\omega\left\langle\Delta \omega_{0}\right\rangle\left(1+k v_{z 0} / \omega\right)\right\}-$ $\left(3 b_{1} \alpha_{0} / 4 \gamma_{10}^{2}-2\left\langle\Delta \omega_{0}\right\rangle^{2} / s E_{0}^{2}\right) A_{0} I_{s} E_{0}$; $\underline{\mathrm{C}}=\left[\Delta \omega_{p \varepsilon}^{2} /\left(1+\varepsilon_{r}\right) \gamma_{10}\right]\left[\left(1+k v_{z 0} / \omega\right)-1 / 2\left(\Omega_{0} \alpha_{0} / \gamma_{10} k c\right)^{2}\right], \quad a_{0}=-$ $\left[\Delta \omega_{p \varepsilon}^{2} \Omega_{0}^{2}\left\langle\Delta \omega_{0}\right\rangle \alpha_{0}^{2} /\left(1+\varepsilon_{r}\right) \gamma_{10}^{3} \omega\right]$, and $\Delta \omega_{p \varepsilon}^{2}=\Delta n_{\varepsilon} e^{2} / m \varepsilon_{0}$. In essence, Eq. (C11) is a fifth order ordinary differential equation (ODE). It is linearized to obtain the relation $\left|\left\langle\Delta \omega_{0}\right\rangle\right|^{3}=\Delta \omega_{p \varepsilon}^{2} \Omega_{0}^{2} \alpha_{0}^{2} / 16\left(1+\varepsilon_{r}\right) \gamma_{10}^{3} \omega \quad$ for determining $\left|\left\langle\Delta \omega_{0}\right\rangle\right|$.

$$
\begin{aligned}
d v_{z} / d t & =d^{2} z / d t^{2}=e v_{\theta} B_{g r} / m=-\partial\left(\mu B_{g z}\right) / \partial z \\
& =-e B_{0} z r v_{\theta} / m L^{2}=-\mu B_{0} z / m L^{2}=-\omega_{b}^{2} z
\end{aligned}
$$

where $\mu=m v_{\theta 0}^{2} / 2 B_{0}$ is the magnetic dipole moment of the electron; $\quad v_{\theta 0}=v_{\theta}(z=0)$ and $B_{g z} \cong B_{0}$ is assumed; $r=r_{L}=\left(v_{\theta 0} / v_{\theta}\right) r_{L 0}$ is equal to the Larmour radius; $r_{L 0}=m v_{\theta 0} / e B_{0}$ is the electron Larmour radius at equator; and $\omega_{b}=\left(\mu B_{0} / \mathrm{mL}^{2}\right)^{1 / 2}$ is the bounce frequency and is assumed to be a constant. Thus a parabolic potential may be used to simulate the mirroring effect of a magnetic dipole field. The approximations that $\omega_{b}=$ const. and $B_{g z} \cong B_{0}$ are justified as long as the magnetic moment $\mu$ varies slowly in time, and $z^{2} / L^{2}$ and $r^{2} / L^{2} \ll 1$.

Acknowledgements. The author is grateful to James T. Huynh, Steven S. Kuo, and Paul Kossey for collaborative works.

This work was supported by the High Frequency Active Auroral Research Program (HAARP), Air Force Research Laboratory at Hanscom AFB, Massachusetts, and by the Office of Naval Research, Grant No. ONR-N00014-05-1-0109. Part of the financial support was arranged through NorthWest Research Associates, Inc.

Edited by: L. Zelenyi

Reviewed by: A. G. Demekhov, L. Stenflo and another anonymous referee

\section{References}

Albert, J. M.: Gyroresonant interactions of radiation belt particles with a monochromatic electromagnetic wave, J. Geophys. Res., 105, 21 191-21 205, 2000.

Arnoldy, R. L. and Kintner, P. M.: Rocket observations of the precipitation of electrons by ground VLF transmitters, J. Geophys. Res., 94, 6825-6832, 1989.

Breneman, A., Kletzing, C. A., Chum, J., Santolik, O., Gurnett, D., and Pickett, J.: Multispacecraft observations of chorus dispersion and source location, J. Geophys. Res., 112, A05221, doi:10.1029/2006JA012058, 2007.

Burgess, W. C. and Inan, U. S.: Simultaneous disturbance of conjugate ionospheric regions in association with individual lightning flashes, Geophys. Res. Lett., 17, 259-262, 1990. 
Cattell, C., Wygant, J. R., Goetz, K., Kersten, K., Kellogg, P. J., von Rosenvinge, T., Bale, S. D., Roth, I., Temerin, M., Hudson, M. K., Mewaldt, R. A., Wiedenbeck, M., Maksimovic, M., Ergun, R., Acuna, M., and Russell, C. T.: Discovery of very large amplitude whistler-mode waves in Earth's radiation belts, Geophys. Res. Lett. 35, L01105, doi:10.1029/2007GL032009, 2008.

Faith, J., Kuo, S. P., and Huang, J.: Chaotic electron motion driven by whistler waves in the magnetosphere, Comments Plasma Phys. Contr. F., 17, 173-182, 1996.

Faith, J., Kuo, S. P., and Huang, J.: Electron precipition caused by chaotic motion in the magnetosphere due to large amplitude whistler waves, J. Geophys. Res., 102, 2233-2241, 1997.

Faith, J., Kuo, S. P., Huang, J., and Schmidt, G.: Precipitation of magnetospheric electrons caused by relativistic effect enhanced chaotic motion in the whistler wave fields, J. Geophys. Res., 102, 9631-9638, 1997.

Helliwell, R. A., Katsufrakis, J. P., and Trimpi, M. L.: Whistler induced amplitude perturbation in VLF propagation, J. Geophys. Res., 78, 4679-4688, 1973.

Helliwell, R. A., Carpenter, D. L., and Miller, T. R.: Power threshold for growth of coherent VLF signals in the magnetosphere, J. Geophys. Res., 85, 3360-3366, 1980.

Helliwell, R. A. and Inan, U. S.: VLF wave growth and discrete emission triggering in the magnetosphere: A feedback model, J. Geophys. Res., 87, 3537-3550, 1982.

Helliwell, R. A.: Controlled stimulation of VLF emissions from Siple Station, Antarctica, Radio Sci., 6, 801-814, 1983.

Ho, A. Y., Kuo, S. P., and Schmidt, G.: Chaotic proton motion driven by kinetic Alfven waves in the magnetosphere leading to polar and equatorial proton precipitations, J. Geophys. Res., 99, 11 087-11 093, 1994.

Imhof, W. L., Robinson, R. M., Colin, H. L., Wygant, J. R., and Anderson, R. R.: Simultaneous measurements of waves and precipitating electrons near the equator in the outer radiation belt, $\mathrm{J}$. Geophys. Res., 99, 2415-2427, 1994.

Kennel, C. F. and Petschek, H. E.: Limit on stably trapped particle flux, J. Geophys. Res., 71, 1-28, 1966.

Khazanov, G. V., Tel'nikhin, A. A., and Kronberg, T. K.: Dynamic theory of relativistic electrons stochastic heating by whistler mode waves with application to the Earth magnetosphere, J. Geophys. Res., 113, A03207, doi:10.1029/2007JA012488, 2008.

Kuo, S. P. and Cheo, B. R.: Relativistic adiabatic invariants of electron motion under ECRH, Phys. Lett. A, 109, 39-42, 1985.
Kuo, S. P. and Lee, M. C.: Saturation of cyclotron maser instability driven by an electron loss-cone distribution, Int. J. Infrared Mill., 7, 623-633, 1986.

Kuo, S. P., Kossey, P., Huynh, J., and Kuo, S. S.: Amplification of whistler waves for the precipitation of trapped relativistic electrons in the magnetosphere, IEEE Trans. Plasma Sci., 32, 362369, 2004.

Kuo, S. P., Kuo, S. S., Huynh, J. T., and Kossey, P.: Precipitation of trapped relativistic electrons by amplified whistler waves in the Magnetosphere, Phys. Plasmas, 14, 009706(1-7), 2007.

Nunn, D., Omura, Y., Matsumoto, H., Nagano, I., and Yagitani, S.: The numerical simulation of VLF chorus and discrete emissions observed on the Geotail satellite using a Vlasov code, J. Geophys. Res., 102, 27 083-27 097, 1997.

Pasmanik, D. L., Titova, E. E., Demekhov, A. G., Trakhtengerts, V., Santolik., O., Jiricek, F., Kudela, K., and Parrot, M.: Quasiperiodic ELF/VLF wave emissions in the Earth's magnetosphere: comparison of satellite observations and modeling, Ann. Geophys., 22, 4351-4361, 2004, http://www.ann-geophys.net/22/4351/2004/.

Pradipta, R., Labno, A., Lee, M. C., Burke, W. J., Cohen, J. A., Burton, L. M., Kuo, S. P., and Rokusek, D.: Electron precipitation from the inner radiation belt above Arecibo, Geophys. Res. Lett., 34, L08101, doi:10.1029/2007GL029807, 2007.

Sazhin, S. S. and Hayakawa, M.: Magnetospheric chorus emissions: A review, Planet. Space Sci., 40, 681-697, 1992.

Trakhtengerts, V.: A generation mechanism for chorus emission, Ann. Geophys., 17, 95-100, 1999, http://www.ann-geophys.net/17/95/1999/.

Trakhtengerts, V., Demekhov, A. G., Titova, E. E., Kozelov, B. V., Santolik., O., Gurnett, D. A., and Parrot, M.: Interpretation of Cluster data on chorus emissions using the backward wave oscillator model, Phys. Plasmas, 11, 1345-1351, 2004.

Tsurutani, B. T. and Smith, E. J.: Postmidnight chorus: a substorm phenomenon, J. Geophys. Res., 79, 118-127, 1974.

Tsytovich, V. N. and Stenflo, L.: Electromagnetic wave instability in a plasma with relativistic particles in a random magnetic field, Astrophys. Space Sci., 89, 233-235, 1983.

Villalon, E. and Burke, W. J.: Near-equatorial pitch angle diffusion of energetic electrons by oblique whistler waves, J. Geophys. Res., 96, 9655-9667, 1991.

Voss, H. D., Imhof, W. L., Mobilia, J., Gaines, E. E., Walt, M., Inan, U. S., Helliwell, R. A., Carpenter, D. L., Katsufrakis, J. P., and Chang, H. C.: Lightning-induced electron precipitation, Nature, 312(20/27), 740-742, 1984. 